\title{
Hemoperitoneo secundario a diverticulitis del sigmoides
}

\author{
Ricardo A. Torres-Rincón', Carolina Salinas-Parra²
}

\section{RESUMEN}

La enfermedad diverticular colónica es la presencia de un divertículo no inflamado en el colon. Se considera una enfermedad común, que se caracteriza por la formación de divertículos falsos en la pared colónica en los puntos de debilidad de esta. Su prevalencia aumenta con la edad y una minoría de los pacientes afectados por esta entidad desarrollará un cuadro de diverticulitis aguda. Sus complicaciones abarcan desde un absceso o flegmón, pasa por la formación de fístulas hasta la obstrucción intestinal y peritonitis. Sin embargo, el desarrollo de un hemoperitoneo espontáneo secundario a una enfermedad diverticular aguda es una forma de presentación inusual y, por tanto, poco reportado en la literatura. Se presenta el caso de un paciente con hemoperitoneo secundario a la perforación diverticular colónica.

\section{PALABRAS CLAVE}

Diverticulitis Colónica; Hemoperitoneo; Perforación IntestinaI; Ruptura Espontánea

\section{SUMMARY}

\section{Hemoperitoneum secondary to sigmoid diverticulitis}

Colonic diverticular disease is the term to describe the presence of an uninflamed diverticulum of the colon. It is considered a common disease, characterized by the formation of false

1 Médico, Cirujano general, Universidad de Antioquía. Hospital Pablo Tobón Uribe, Medellín, Colombia.

2 Médica, Cirujana general, Universidad Pontificia Bolivariana, Medellín, Colombia.

Correspondencia: Ricardo Andrés Torres-Rincón; riandrestorresr@hotmail.com

Recibido: mayo 14 de 2018

Aceptado: septiembre 06 de 2018

Cómo citar: Torres-Rincón RA, Salinas-Parra C. Hemoperitoneo secundario a diverticulitis del sigmoides. latreia. 2019 Abr-Jun; 32(2):141-146. D0I 10.17533/udea.iatreia.01. 
diverticula in the colonic wall at points of weakness of that, its prevalence increases with age and a minority of patients affected by this disease develops acute diverticulitis. Its complications range from abscess or phlegmon, fistula formation, to intestinal obstruction and peritonitis. However, the development of spontaneous hemoperitoneum secondary to acute diverticular disease is an unusual form of presentation and therefore scarcely reported in the literature. Here is presented a case with a hemoperitoneum secondary to colonic diverticular perforation.

\section{KEY WORDS}

Diverticulitis, Colonic; Hemoperitoneum; IntestinaI Perforation; Rupture, Spontaneous

\section{INTRODUCCIÓN}

La diverticulosis es una enfermedad común en los países occidentales y desarrollados. Es una condición que aumenta su prevalencia con la edad y es similar entre hombres y mujeres. Su fisiopatología está explicada por el aumento de la presión intracolónica por factores de riesgo modificables como la obesidad, la actividad física y la dieta, que determinan el esfuerzo con que se realiza la deposición por heces duras; $y$ factores no modificables como el debilitamiento de la matriz extracelular de la pared del colon, relacionado con los cambios de elastina y colágeno secundario a la edad (1). Esto genera la formación de pseudodivertículos en el colon en sitios donde la vasculatura penetra la pared intestinal en la capa muscular circular (2). Estos divertículos se localizan principalmente en el colon descendente distal y siogmoides, sin compromiso del recto (1).

La mayoría de los pacientes con diverticulosis permanecen asintomáticos, el 20 \% desarrollan síntomas (enfermedad diverticular/diverticulitis) y solo el $15 \%$ de estos tendrán complicaciones (3).

Los casos complicados de diverticulitis pueden presentarse como abscesos locales, peritonitis, formación de fístulas, obstrucción intestinal o abscesos hepáticos, siendo la más común la formación de abscesos locales en un $16 \%$ de los pacientes con diverticulitis aguda, las fístulas en un $12 \%$ y las perforaciones/peritonitis ocurren en 1-2\%, con la más alta mortalidad (20\%) (4).
El hemoperitoneo espontáneo, definido como el sangrado en la cavidad abdominal de origen no traumático, es una presentación inusual de múltiples enfermedades y condiciones, de las cuales la más común es la casusa ginecológica (5). Predomina en pacientes con trastornos de la coagulación y, debido a lo raro de su presentación, el diagnóstico solo se hace una vez se han realizado imágenes diagnósticas o el paciente ha sido llevado a cirugía (6).

No existe mención clara, en los artículos de revisión de hemoperitoneo espontáneo, que establezca como posible causa un origen colónico (6). $\mathrm{Y}$ posterior a una revisión de la literatura se encontraron pocos casos reportados al respecto, razón por la que se presenta este caso de un paciente con hemoperitoneo espontáneo secundario a una diverticulitis complicada del sigmoides.

\section{PRESENTACIÓN DEL CASO}

Paciente de sexo masculino, 74 años, hipertenso, sin antecedentes quirúrgicos abdominales, ingresa al servicio de urgencias porque presenta 20 días de dolor abdominal generalizado de predominio en fosa iliaca izquierda, asociado a primer episodio de melenas, sin otros síntomas gastrointestinales. Clínicamente con abdomen distendido, dolor moderado generalizado, a la palpación predominantemente en hemiabdomen izquierdo, sin signos de irritación peritoneal. Signos vitales estables. Paraclínicos con creatinina $1,2 \mathrm{mg} /$ dL, hemoglobina 12,6 gr/dL, leucocitos 19.500 células/ $\mathrm{mm}^{3}$, neutrófilos 18.100 células $/ \mathrm{mm}^{3}$, plaquetas 50.000 células $/ \mathrm{mm}^{3}$. Se realiza una tomografía de abdomen contrastada que reporta plastrón inflamatorio en el colon, en la unión del colon descendente y el sigmoides, importante congestión vascular y estriación de la grasa, con engrosamiento parietal concéntrico de la pared, en el meso del colon siogmoides con una imagen sugestiva de hematoma con sangrado activo, líquido libre en cantidad sionificativa en todos los recesos abdominales, de alta atenuación, por posible contenido hemorrágico, no aire libre ni extravasación del medio de contraste oral. Bazo aumentado de tamaño, con un diámetro de $18 \mathrm{~cm}$, sin lesiones focales (Figura 1. A y B). Se piensa en realizar un manejo con arteriografía y embolización, pero el paciente presenta descenso de HB a 9,2 y plaquetas en 27.000 en 24 
horas. Hay un aumento del dolor abdominal sin descartarse una peritonitis purulenta o fecal, por lo que se lleva a laparotomía exploratoria. Se documenta hemoperitoneo de 3,5 litros, hepatoesplenomegalia, plastrón de epiplón que involucra un divertículo en el borde antimesentérico del colon sigmoides, sin evidencia de perforación macroscópica: no líquido libre peritoneal fecal ni purulento (Figura 2).
A

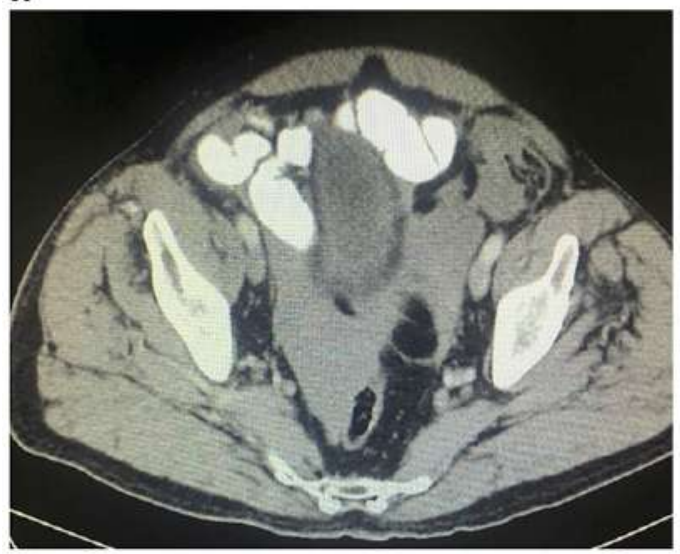

B

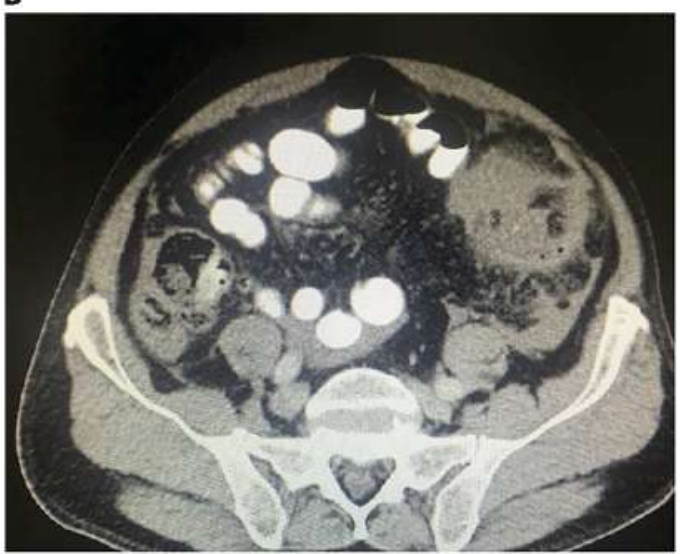

Figura 1. Tomografía contrastada de abdomen. A. Presencia de líquido libre en cavidad abdominal de alta densidad: hemoperitoneo. B. Plastrón inflamatorio en fosa iliaca izquierda asociado con una enfermedad diverticular del colon. Fuente: propia.

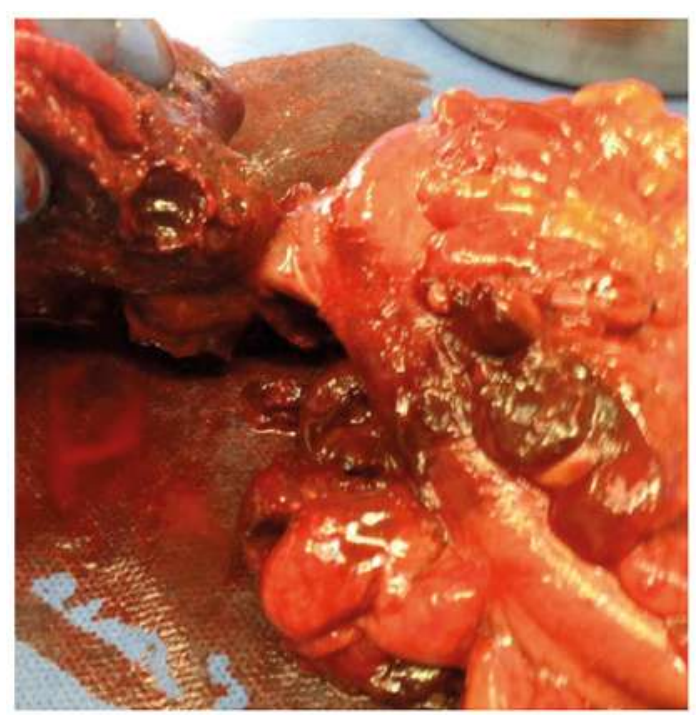

Figura 2. Pieza quirúrgica. Colon sigmoides con divertículo antimesentérico y un gran hematoma mural. Fuente propia.
Se realiza el drenaje del hemoperitoneo, siomoidectomía que deja los extremos intestinales cerrados. Empaquetamiento abdominal del flanco izquierdo por coagulopatía y se decide dejar con el abdomen abierto, con el cierre temporal de la pared abdominal. El paciente nuevamente es intervenido a las 72 horas, una vez mejoraron sus condiciones hemodinámicas. Se realiza el desempaquetamiento abdominal, colostomía tipo Hartmann del colon descendente y el cierre definitivo de la pared abdominal. El paciente presenta un ileo postoperatorio prolongado que requirió el inicio nutrición parenteral total. Se inició tratamiento antibiótico por bacteriemia asociada al catéter venoso central por Pseudomonas Aeruginosa. Por el hallazgo al ingreso de trombocitopenia se realiza biopsia hepática que reporta esteatohepatitis yse descartaron enfermedades infecciosas. Se realizó el aspirado de la médula ósea con médula hematopoyética normal, sin cambios significativos en la citometría de flujo y 3 líneas celulares normales. Debido a la persistencia de la trombocitopenia, a pesar de la resolución del sanorado intraabdominal agudo y el 
proceso infeccioso, se realiza diagnóstico de púrpura trombocitopénica idiopática sin necesidad de iniciar manejo con esteroides. Finalmente egresa del hospital con conteo de plaquetas mayor de 50.000/L. En el seguimiento ambulatorio el paciente presenta buena evolución. La patología quirúrogica reporta pseudodivertículos del colon y un hematoma abscedado pericolónico, se descarta la presencia de un tumor estromal gastrointestinal por inmuno histoquímica.

\section{DISCUSIÓN}

Los divertículos son alteraciones en la estructura del colon que se visualizan como "sacos", se generan por herniación de la mucosa y la submucosa a través de la capa muscular circular de la pared intestinal (pseudodiverticulos o divertículos falsos). Estas prominencias saculares se desarrollan en sitios de mayor debilidad de la pared, que coincide con la entrada de los vasos rectos a la pared (4). Pueden existir divertículos verdaderos del colon (contienen las tres capas de la pared intestinal), pero estos son menos comunes y son más prevalente en el colon derecho en poblaciones de Asia. La diverticulosis es sinónimo de enfermedad diverticular, hace referencia a la presencia de uno o más divertículos en la pared del colon (7). La inflamación de estos divertículos se define como diverticulitis, que comúnmente se acompaña de perforación micro o macroscópica (2).

La prevalencia general de la enfermedad diverticular varía considerablemente con la edad, se plantea que aproximadamente el $5 \%$ de los menores de 40 años tienen diverticulosis (7) y aumenta exponencialmente. De esta manera, el 50\% de los pacientes mayores de 60 años y el 70\% de los pacientes mayores de 80 años presentan enfermedad diverticular. Debido a su alta prevalencia se considera una de las causas más comunes de hospitalización relacionada al tracto gastrointestinal y es la anormalidad más frecuentemente encontrada en una colonoscopia (4). La colonoscopia es la herramienta diagnóstica considerada de elección para su abordaje inicial; sin embargo, es usualmente evitada en casos de presencia de diverticulitis, por el riesgo de perforación por la insuflación de aire o durante la manipulación del colonoscopio. En casos agudos puede ser únicamente útil ante la presencia de imágenes equívocas para diferenciar otras enfermedades inflamatorias que pueden coexistir con la diverticulosis (colitis, cáncer, enfermedad inflamatoria intestinal) (8). Una vez se resuelva el episodio agudo se recomienda realizar una colonoscopia para excluir la posibilidad de una neoplasia colónica subyacente (8). La imagen que se considera de elección en el episodio agudo de inflamación diverticular es la tomografía computarizada, que brinda una sensibilidad de 93-97 \% y una especificidad del $100 \%$ para su diagnóstico (2).

La mayoría de los pacientes tendrán un curso asintomático, del 10-25\% presentarán un episodio de diverticulitis en algún momento de su vida (9) y solo el $4 \%$ del total de los pacientes con diverticulosis presentarán alguna complicación. Solo el $1 \%$ requerirá un procedimiento quirúrơico y 1-2 \% tendrán una perforación libre (2). El $80 \%$ de los pacientes con diverticulitis perforada no han tenido historia previa de enfermedad diverticular (8). El mayor temor de la enfermedad diverticular es la recurrencia de los síntomas; se reporta una incidencia del $15-20 \%$ a 5 años después de un primer episodio de diverticulitis aguda, con un riesgo muy bajo de requerir cirugía emeroente $(<5 \%)(10)$.

Solo el $15 \%$ de los divertículos afectan el colon derecho, los divertículos gigantes se denominan a aquellos que miden más de $4 \mathrm{~cm}$, con tamaños máximos reportados de $25-30 \mathrm{~cm}$, se desarrollan principalmente en el sigmoides y tienen como complicación específica el vólvulus $(11,12)$.

En países occidentales el 90 \% de la enfermedad diverticular compromete al colon izquierdo y el $99 \%$ tienen algún compromiso del sigmoides (9). En esta localización son catalogados como divertículos por pulsión, donde su desarrollo depende de dos factores principales: el aumento de la presión intraluminal y la presencia de puntos de debilidad en la pared intestinal (7). La presión intraluminal aumentada está determinada por factores de riesgo como las alteraciones en la motilidad, alteraciones anatómicas propias del componente muscular del colon izquierdo (acortamiento y engrosamiento de la muscular propia) (7), inactividad física, obesidad, tabaquismo, constipación, uso de antiinflamatorios no esteroideos (2). Los puntos de debilidad de la pared del colon están dados principalmente por las diferencias que tiene el colon con el intestino delgado y el recto, los cuales se encuentran revestidos por dos capas musculares (circular y longitudinal), en contraposición con el colon que solo está cubierto por una sola capa 
muscular circular, ya que la capa longitudinal externa se condensa en lo que se conoce como la taenia coli. Adicionalmente a esto, la penetración de los vasos mesentéricos en dos puntos en la capa muscular, a cada lado de la pared, convierten dichos puntos en sitios vulnerables para la herniación de la mucosa y la submucosa (7).

La dieta alta en fibra se ha demostrado que no previene el desarrollo de divertículos, pero puede prevenir la diverticulitis (4). Las potenciales complicaciones de la diverticulitis son la formación de abscesos, fístulas, obstrucción intestinal y perforación-peritonitis, en orden decreciente de frecuencia (4).

EI hemoperitoneo espontáneo es una condición catastrófica que se define como el sangrado en la cavidad peritoneal de origen no traumático, puede ser idiopático o tener un origen claro: hepático, esplénico, vascular arterial y venoso, trastornos de la coagulación o ginecológico, es esta última la más frecuentemente reportada. Su estudio inicial de elección es la tomografía computarizada que hará el diaonóstico inicial de sangre en la cavidad abdominal y estudiará la posible causa subyacente (13). La ecografía puede ser una herramienta de enfoque inicial, pero no determina la presencia de sangrado activo ni la causa de base. Su manejo será determinado por la estabilidad hemodinámica del paciente, son opciones la arteriografía y la embolización como la cirugía emergente $(6,14)$.

La apoplejía abdominal es el sangrado peritoneal espontáneo, idiopático (sin un factor predisponente claro). Puede ser intra o retroperitoneal, se asocia frecuentemente a hipertensión (33-50 \%) y ateroesclerosis (80-87\%) (15). Descrita por primera vez en 1909, y dado lo raro de esta presentación, la tomografía computarizada no es tan sensible para realizar el diagnóstico, y en este caso la cirugía tiene un papel primordial (16).

En las revisiones de sangrado espontáneo no existe la inclusión de la diverticulitis como posible causa. Al realizar la revisión de la literatura, existen a la fecha cuatro artículos (5, 17-19), que similar al caso que se expuso en esta revisión, presentaron hemoperitoneo secundario a la perforación de un divertículo en el colon sigmoides. Dado lo inusual de esta manifestación como complicación de la diverticulitis y lo importante de tenerla en cuenta en la evaluación de estos pacientes, se muestra este caso. No existe en la literatura alguna asociación de la púrpura trombocitopenia idiopática con este tipo de síntoma de la enfermedad diverticular, sin embargo, claramente la trombocitopenia expuso al paciente al riesgo del sangrado abdominal mayor.

\section{CONFLICTOS DE INTERESES}

Se obtuvo el consentimiento informado por escrito del paciente para la elaboración de este manuscrito y de las imágenes que lo acompañan. Del mismo modo, se declara que se han seguido los protocolos establecidos por el Hospital donde ocurrió para acceder a los datos de la historia clínica para realizar esta publicación, con la finalidad de generar investigación y divulgación (investigación y divulgación) en la comunidad científica. Se informa que no existe relación financiera o personal de ninguno de los autores de la publicación que genere conflicto de intereses.

\section{REFERENCIAS BIBLIOGRÁFICAS}

1. West AB; NDSG. The pathology of diverticulitis. J Clin Gastroenterol. 2008 Nov-Dec;42(10):1137-8. DOI 10.1097/MCG.0b013e3181862a9f.

2. Jacobs DO. Clinical practice. Diverticulitis. N EngI J Med. 2007 Nov;357(20):2057-66.

3. Tursi A. New medical strategies for the management of acute diverticulitis. Expert Rev Gastroenterol Hepatol. 2015;9(10):1293-304. DOI 10.1586/17474124.2015.1075881.

4. Feuerstein JD, Falchuk KR. Diverticulosis and Diverticulitis. Mayo Clin Proc. 2016 Aug;91(8):1094-104. DOI 10.1016/j.mayocp.2016.03.012.

5. George J, Ben-Sassi A, Dixon R. Spontaneous haemoperitoneum due to a sigmoid diverticulum. BMJ Case Rep. 2014 Dec;2014. pii: bcr2014207849. DOI 10.1136/ bcr-2014-207849.

6. Kasotakis G. Spontaneous hemoperitoneum. Surg Clin North Am. 2014 Feb;94(1):65-9. DOI 10.1016/j. suc.2013.10.006.

7. Brian West A. The pathology of diverticulosis: classical concepts and mucosal changes in diverticula. J Clin Gastroenterol. 2006 Aug;40 SuppI 3:S126-31. 
8. Stollman N, Smalley W, Hirano I; AGA Institute Clinical Guidelines Committee. American Gastroenterological Association Institute Guideline on the Management of Acute Diverticulitis. Gastroenterology. 2015 Dec;149(7):1944-9. DOI 10.1053/j.gastro.2015.10.003.

9. Vermeulen J, van der Harst E, Lange JF. Pathophysiology and prevention of diverticulitis and perforation. Neth J Med. 2010 Oct;68(10):303-9.

10. Shah SD, Cifu AS. Management of Acute Diverticulitis. JAMA. 2017 JuI;318(3):291-2. DOI 10.1001/ jama.2017.6373.

11. Stollman N, Raskin JB. Diverticular disease of the colon. Lancet. 2004 Feb 21;363(9409):631-9.

12. Chater C, Saudemont A, Zerbib P. Giant colon diverticulum. J Visc Surg. 2015 Nov;152(5):336-8. DOI 10.1016/j.jviscsurg.2015.06.002.

13. Lucey BC, Varghese JC, Anderson SW, Soto JA. Spontaneous hemoperitoneum: a bloody mess. Emero Radiol. 2007 Jun;14(2):65-75.
14. Lucey BC, Varghese JC, Soto JA. Spontaneous hemoperitoneum: causes and significance. Curr Probl Diagn Radiol. 2005 Sep-Oct;34(5):182-95.

15. Cawyer JC, Stone CK. Abdominal apoplexy: a case report and review. J Emerg Med. 2011 Mar;40(3):e49-52. DOI 10.1016/j.jemermed.2007.11.080.

16. Reilly EF, Blansfield JA, Zebley DM. Spontaneous coIonic mesenteric hemorrhage: report of an unusual case of abdominal apoplexy. Dis Colon Rectum. 2005 Jul;48(7):1484-6.

17. Lawson HH. Haemoperitoneum associated with a solitary diverticulum of the siogmoid colon. S Afr Med J. 1961 Aug;35:715-6.

18. Sancho C, Garcia A, García MJ, Villalba F. Hemoperitoneo por divertículo solitario de sigma Liposarcoma mixoide retroperitoneal con metaplasia ósea. Cir Esp. 2007;81(5):290-1. DOI 10.1016/S0009-739X(07)71325-8.

19. Kye BH, Kim HJ, Yoon JH, Park DC, Lee SJ. Spontaneous hemoperitoneum caused by a diverticulum of the siogmoid colon. Int J Med Sci. 2011;8(6):467-9. 